\title{
Drosophila huancavilcae: ciclo biológico y cariotipo metafásico
}

Ana Beatriz Mafla M.

Laboratorio de Genética Evolutiva. Escuela de Ciencias Biológicas. Pontificia Universidad Católica del Ecuador. Apartado 17-01-2184, Quito, Ecuador, (amafla @puce.edu.ec).

RESUMEN. Se reportan los datos del ciclo biológico y el cariotipo mitótico de Drosophila huancavilcae; hay mucha semejanza con las especies $D$. inca y $D$. yangana, con las cuales conforma el subgrupo inca.

PALABRAS CLAVE. Drosophila huancavilcae, ciclo de vida, cariotipo.

ABSTRACT . The data of the biological cycle and metaphasic karyotype of Drosophila huancavilcae are reported; there is much similarity with species D. yangana and D. inca, with who constitute the inca subgroup.

KEYWORDS: Drosophila huancavilcae, life cycles, karyotype.

\section{INTRODUCCIÓN}

Drosophila huancavilcae Rafael \& Arcos, 1989; es una especie descubierta en Ecuador que, junto a $D$. inca Dobzhansky \& Pavan, 1943 y D. yangana Rafael \& Vela, 2003, forma el subgrupo inca del grupo repleta, que fuera establecido en 1989 por Rafael y Arcos. $(1,2)$

Con el objeto de caracterizar a estas especies se ha estudiado sus ciclos biológicos y sus cromosomas metafásicos, de tal manera que conocemos aspectos básicos de la biología de $D$. inca y D. yangana. $(3,4)$ En este trabajo, se reporta el ciclo de vida, los valores de aptitud y se describe el cariotipo metafásico de Drosophila huancavilcae utilizando la nomenclatura cromosómica recomendada por Levan et al. (5)

\section{MATERIALES Y MÉTODOS}

En mayo 2005 se realizó una prospección de la drosofauna en la provincia de Manabí, Ecuador. En la localidad de Montecristi (1 $1^{\circ} 2$ ' $46^{\prime \prime} \mathrm{S} 80^{\circ} \quad 40^{\prime} 44^{\prime \prime} \mathrm{W}$ $450 \mathrm{~m}$ ) se recolectaron 24 hembras y 23 machos de $D$. huancavilcae que fueron multiplicados durante un año. Los datos del ciclo biológico, obtenidos en el laboratorio, corresponden a unas 15 generaciones mantenidas entre mayo del 2005 a junio del 2006 y procedentes de 712 siembras realizadas en tubos de ensayo con el medio de cultivo descrito por Rafael et al. (6), la temperatura ambiental se mantuvo en un estrecho margen de $21^{\circ} \mathrm{C}$ a $23^{\circ} \mathrm{C}$, con períodos de luz: oscuridad (12:12). 
Se midieron diez huevos de $D$. huancavilcae; para ello se usó un microscopio Zeiss, objetivo $40 \mathrm{X}$ y una laminilla con escala micrométrica.

Dos o tres veces por semana se hicieron registros de las fechas de aparecimiento de huevos, y de cada fase de desarrollo hasta el momento de emergencia de adultos.

En la descripción del ciclo se hace referencia al Modo (moda o número modal) como un parámetro adecuado; pero también se dan los valores medios y las desviaciones estándar en días de duración de cada fase de desarrollo. Se presentan los componentes de la aptitud que son: la fertilidad definida como el número de descendientes producido (7); tal valor se expresa en número de tubos conteniendo huevos por número de tubos sembrados $(\mathrm{H} / \mathrm{S})$ y se transforma a porcentaje. La viabilidad expresada en número de tubos en los que emergieron adultos por el número de tubos que contenían huevos $(\mathrm{A} / \mathrm{H})$ e igualmente transformada en porcentaje; la madurez del adulto: tiempo promedio, en días, que demora el adulto para cruzarse y dejar descendencia y la velocidad de desarrollo expresada en promedio de días de duración desde la eclosión de la larva hasta la emergencia del imago.

Para el análisis citológico, se disectaron larvas de tercer estadio para extraer los ganglios cerebrales; en un portaobjeto excavado se colectaron de 10 a 12 ganglios en solución de Ringer-Drosophila, se transfirieron a otro porta excavado conteniendo una solución de Ringer: colchicina $0,01 \mathrm{M}$ en proporción 2:1 por 30 minutos; se extrajo esta solución remplazándola por $\mathrm{KCl}$ 0,075M durante 20 minutos. Después del tratamiento hipotónico se fijó el tejido en metanol: ácido acético (3:1) por mínimo 30 minutos, luego se extrajo el fijador y se dispersó el tejido en ácido acético $60 \%$, se añadió dos gotas de fijador y se goteó en portaobjetos previamente mantenidos en etanol y en refrigeración; las placas con la suspensión se dejaron secar en una plancheta a $65^{\circ}$ C (el choque térmico permite una mejor dispersión de los cromosomas). Las placas fueron teñidas con Giemsa diluido en agua desionizada en proporción 1:20.

Los mejores núcleos fueron fotografiados en un fotomicroscopio Zeiss usando el lente de inmersión Neofluar 100, oculares $8 \mathrm{X}$ y aumento 1,25 de la cámara fotográfica obteniéndose una amplificación total de $1000 X$.

Los negativos fueron reproducidos con un scanner Photosmart HP Scanjet 4070, con el objeto de realizar los cariotipos en fotografías digitales.

Se midieron los cromosomas y se organizaron en orden descendente; usando el programa Photoshop. Se dispusieron los cromosomas por tamaño decreciente; en la identificación de la forma cromosómica se sigue las recomendaciones de Levan et al. (5), nominando a los cromosomas st, sm o $\mathrm{T}$ de acuerdo a la posición del centrómero en la región subterminal, submedial o en el punto Terminal, respectivamente.

Los idiogramas se construyeron normalizando los valores de 27 cariotipos (18 machos, 9 hembras). La normalización se realizó calculando la longitud relativa de cada cromosoma y luego promediando los valores de cada par homólogo, excepto para el par XY; igual procedimiento se aplicó en la normalización del índice centromérico. 


\section{RESULTADOS Y DISCUSIÓN}

Los datos del ciclo biológico de $D$. huancavilcae así como los valores de aptitud darwiniana, obtenidos en laboratorio, son bastante semejantes a los de las otras especies del subgrupo inca: $D$. inca y $D$. yangana. Los huevos tienen, en promedio, $480 \mu$ de longitud y $183 \mu$ en la parte central más ancha; presentan cuatro filamentos de $565 \mu$ de longitud: dos nacen del extremo anterodorsal y dos emergen aproximadamente a un tercio de la longitud en la superficie ventral. Esta primera fase tiene una duración de tres días (número modal). Al segundo o tercer día de la ovo posición emerge una pequeña larva de cerca de 1 $\mathrm{mm}$ de largo y tiene dos mudas; la primera fase larvaria presenta un número modal de tres días de duración en tanto que la segunda dura 4 y la tercera 6 .

El período de pupación es de siete días.

Tabla 1. Duración en días de cada fase de desarrollo en Drosophila huancavilcae

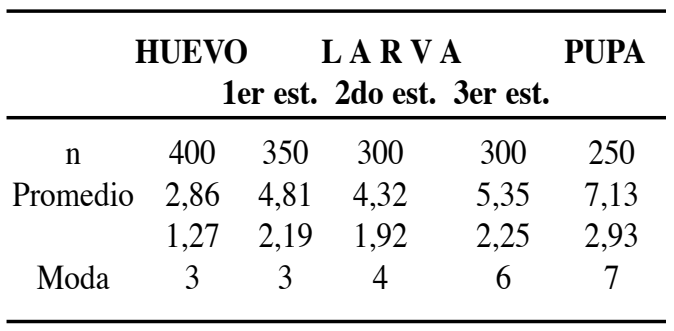

En suma, los huevos de D. huancavilcae son parecidos, en tamaño, a los de $D$. yangana y menores a los de $D$. inca. La duración de las fases del ciclo es más parecida a $D$. inca, salvo en el $2^{\circ}$ estadío larvario que aumenta en 1 día y que afecta por igual a la velocidad de desarrollo que es en promedio de 23,4 días. La maduración de los adultos 5,1 días es muy semejante a las otras especies del subgrupo. Ver Tabla 2, donde se resumen estos valores, así como los de otros componentes de la aptitud encontrados en $D$. huancavilcae: la fertilidad es de $67,97 \%$, la viabilidad de 64,4 $\%$, estas cifras, bastante más bajas que las de $D$. inca y de $D$. yangana, sugieren que hay algún elemento esencial que falta en las condiciones de crianza.

Tabla 2. Componentes de la aptitud de $\boldsymbol{D}$. huancavilcae

\begin{tabular}{lcc}
\hline Fertilidad H/S & $484 / 712$ & $\mathbf{6 7 , 9 7 \%}$ \\
Viabilidad A/H & $310 / 484$ & $\mathbf{6 4 , 4} \%$ \\
Velocidad de desarrollo $(\mathrm{n}=150)$ & $\mathbf{2 3 , 4 7 3 3} \pm 2,6601$ \\
Maduración adultos $(\mathrm{n}=350)$ & $\mathbf{5 , 0 7 7} \pm 1,6831$ \\
\hline
\end{tabular}

H/S Tubos con huevos por tubos con siembras; $\mathrm{A} / \mathrm{H}$ Tubos en los que emergieron adultos por tubos con huevos.

La velocidad de desarrollo y la maduración de adultos en días

Respecto al cariotipo de $D$. huancavilcae sus características son: $2 \mathrm{n}=12$ con 4 pares autosómicos subtelocéntricos, 1 telocéntrico; y el par sexual heteromórfico. (Fig. 1a, b). El grupo A reúne al par autosómico 2 que es el más grande con una longitud relativa (LR) de 11,89; al par 3 de LR 10,08 y al par sexual con el X de LR 9,83 que se confunde con los anteriores y el cromosoma $Y$ que resalta por su heteropicnosis, es subtelocéntrico y de LR 9,31; en los núcleos bien extendidos es observable una constricción secundaria en el brazo largo del Y, lo cual le da semejanza a $D$. yangana.

El grupo B con los 3 autosomas más pequeños (LR: 8,$64 ; 7,41$ y 2,21); reúne a los pares 4 y 5 muy parecidos, difíciles de distinguir y al autosoma 6 claramente iden- 


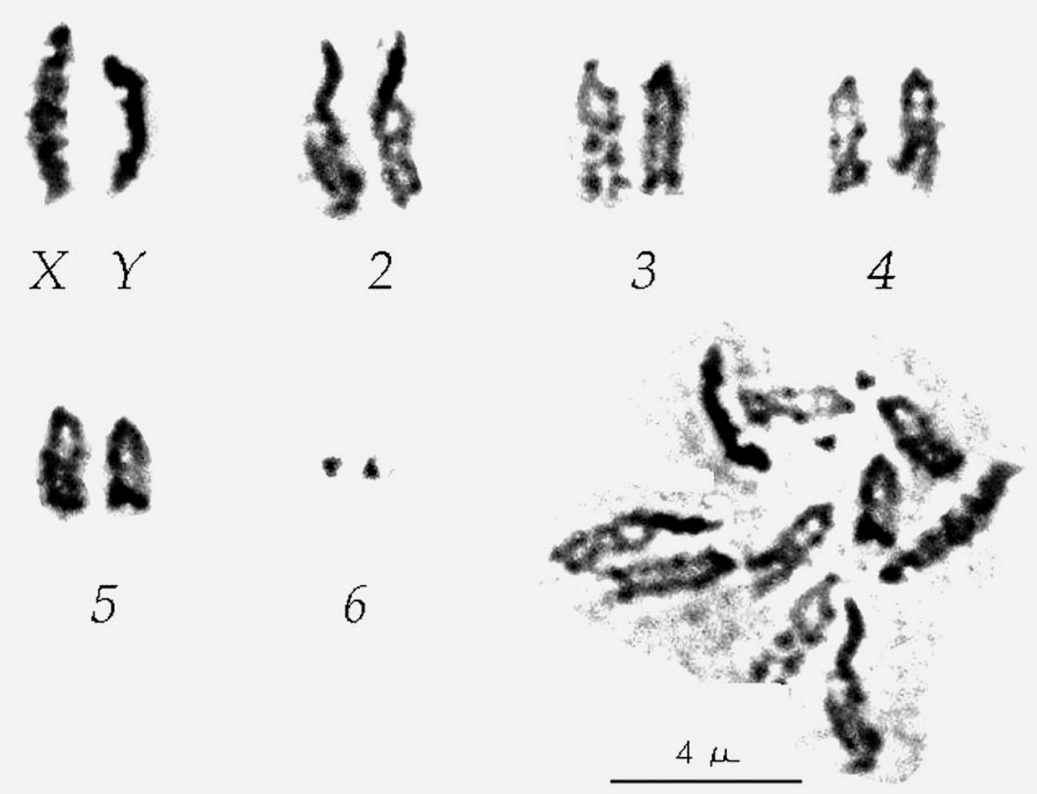

Figura 1. a) Núcleo mitótico de Drosophila huancavilcae macho

b) Cariotipo mostrando los grupos cromosómicos A y B

tificable porque aparece fuertemente heteropicnótico, es telocéntrico o puntiforme (en la nomenclatura clásica).

Se registraron cromosomas supernumerarios en tres cariotipos de los 27.

El idiograma (Figura 2, Tabla 3) resume las características de los cromosomas de esta especie. Lo más notable es el $n=6$

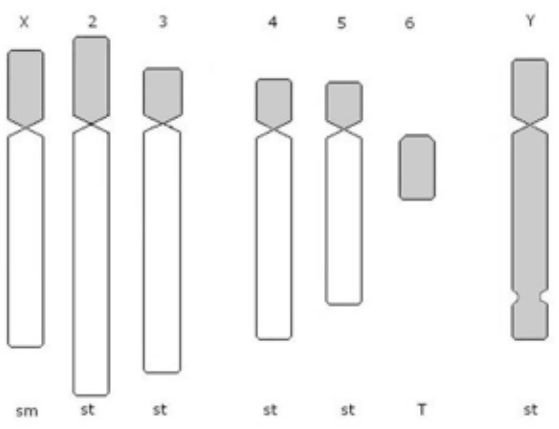

y la presencia del pequeño 6 telocéntrico que nos permite considerar a la especie como una forma primitiva del género. Sin embargo, resulta paradójico el contenido de heterocromatina concentrada en los brazos cortos de todos los cromosomas subtelocéntricos, en la totalidad del cromosoma Y y en el microcromosoma, pues como se conoce por la literatura de Drosophila se cree que las especies que concentran heterocromatina son derivadas. (8)

Ahora que tenemos descritos los cariotipos de las 3 especies resulta interesante comparar los contenidos y distribución de heterocromatina mediante técnicas de bandeo. En forma muy bizarra podemos plantear que la proporción de heterocromatina en orden ascendente va desde $D$. huancavilcae a $D$. yangana y luego a $D$. inca. 
Tabla 3. Longitud relativa, Índice Centromérico, Morfología y Porcentaje de heterocromatina de los cromosomas mitóticos de D. huancavilcae

\begin{tabular}{ccccc}
\hline $\begin{array}{c}\text { Par } \\
\text { Crom. }\end{array}$ & LR & IC & Morfología & $\begin{array}{c}\% \\
\text { heterocromatina }\end{array}$ \\
\hline 2 & 11,89 & 24,23 & st & 5,76 \\
3 & 10,08 & 18,28 & st & 3,68 \\
X & 9,83 & 26,93 & sm & $5,28 *$ \\
4 & 8,64 & 19,69 & st & 3,40 \\
5 & 7,41 & 22,44 & st & 3,32 \\
6 & 2,21 & 0 & T & 4,42 \\
\hline
\end{tabular}

* En machos varía: $11,95=\mathrm{X} 2,64+\mathrm{Y} \quad 9,31$ $\mathrm{n}=27$ cariotipos

\section{AGRADECIMIENTOS}

A Gabriela Romero que trabajó pacientemente en el registro y síntesis de los períodos de duración de las fases de desarrollo y a mi colega Violeta Rafael por sus valiosos comentarios y sugerencias. El presente trabajo es parte del proyecto: "Caracterización de nuevas especies ecuatorianas de Drosophila" financiado por la Pontificia Universidad Católica del Ecuador.

\section{REFERENCIAS BIBLIOGRÁFICAS}

1. RAFAEL, V. \& ARCOS, G. 1989. Subgrupo inca, un nuevo subgrupo del grupo repleta, con descripción de Drosophila huancavilcae nov. sp. (Diptera, Drosophilidae). Evolución Biológica, Bogotá, 3(3): 233 243.

2. RAFAEL, V. \& VELA, D. 2003. Drosophila yangana sp. nov. un nuevo miembro del grupo repleta, subgrupo inca (Diptera: Drosophilidae). Revista de la Pontificia Universidad Católica del Ecuador, Quito, 71:129-139.

3. MAFLA, A. B. 2005 . Ciclos de vida y componentes de la aptitud de Drosophila inca y D. yangana (Diptera, Drosophilidae). Iheringia, Série Zoologia., Porto Alegre, 95 (1): 89-91.

4. MAFLA, A. B.2005. Cariotipos metafásicos de Drosophila inca y D. yangana, subgrupo inca grupo repleta. Revista Ecuatoriana de Medicina y Ciencias Biológicas. Vol XXVII, $N^{\circ} 1$ y 2: 21-25.

5. LEVAN, A., FREDGA, K. \& SANDBERG, A.A. 1964. Nomenclature for centromeric position on chromosomes. Hereditas, 52 (2): 201-220.

6. RAFAEL, V. \& VELA, D. 2000. Drosophila distribution in Ecuador. Drosophila Information Service, Norman, 83:85-88.

7. MATHER, K. 1983. Response to Selection. In: The Genetics and Biology of Drosophila. Edited by: Ashburner, M., Carson, H. L. and Thompson, J. N. Jr. Academic Press. London, 3c: 155-221.

8. YOON, J.S. \& RICHARDSON, R.H. 1978. Evolution in Hawalian Drosophilidae. III. The Microchro-mosome and heterochromatin of Drosophila. Evolution, 32 (3): 475 484 . 